\title{
Convergencia y Estabilidad de Los Tipos De CAmbio Europeos: Una Aplicación DE EXPONENTES DE LYAPUNOV*
}

\author{
Elena Olmedo \\ Universidad de Sevilla \\ RICARDO GIMENO \\ Universidad Pontificia Comillas \\ LORENZO ESCOT \\ Universidad Complutense de Madrid \\ Ruth Mateos \\ Universidad San Pablo-CEU
}

In this paper we applied the dynamic system theory to the measurement of the stability of the European process of convergence. In particular, Lyapunov's exponents are used to verify the influence of political and economic decisions made during the creation of the European Union on the stability (or instability) of exchange rate fluctuation in different European countries. We find evidence of such relationship.

JEL: C45, C61, F02

Keywords: estabilidad, sistemas dinámicos, convergencia europea, euro, exponente de Lyapunov, redes neuronales.

\section{Introducción: Sistemas DinÁmicos y Estabilidad de los Tipos De CAMBio}

Es sobradamente conocido que la volatilidad de los tipos de cambio está caracterizada por una considerable persistencia, de manera que grandes movimientos en los mismos están seguidas de a su vez otros grandes movimientos, existiendo correlación serial positiva en sus cuadrados. Por este motivo, la volatilidad presente y pasada puede utilizarse para predecir la volatilidad futura. En definitiva, se puede considerar que el valor del tipo de cambio en el momento

\footnotetext{
*Email: olmedo@us.es,rgimeno@upco.es, escot@ccee.ucm.es, matcab@ceu.es
} 
presente es una función del valor que presentaba en el pasado, definiéndose así un sistema dinámico.

Una vez enfocado el problema desde este punto de vista, parece lógico pensar que la estabilidad del sistema explicará, en gran medida, la volatilidad observada en el tipo de cambio. Si el sistema dinámico subyacente es inestable el tipo de cambio será también inestable. De esta manera, en el presente trabajo se utiliza una medida de la estabilidad de los sistemas dinámicos para analizar la estabilidad en los tipos de cambio.

¿Cómo se miden las propiedades de estabilidad del sistema dinámico? Si el sistema dinámico es lineal, se trabaja con sus autovalores, distinguiéndose en este caso el comportamiento a largo plazo estable (si el autovalor es de módulo menor que la unidad) o explosivo (si el autovalor es de módulo mayor que la unidad). El problema al que nos enfrentamos, sin embargo, es más complejo debido a que este tipo de sistemas lineales no puede generar un comportamiento volátil en los tipos de cambio, que sea tan complejo como el observado en la realidad. Para ello es necesario introducir no linealidades en el sistema.

Si el sistema dinámico es no lineal, sus propiedades de estabilidad se describen mediante los exponentes de Lyapunov (Lyapunov, 1892): si el máximo exponente de Lyapunov es positivo, el sistema es inestable y si es negativo, el sistema es estable. El sistema será tanto más estable conforme mayor sea, en valor absoluto, el exponente de Lyapunov. Un sistema dinámico se dice que es caótico si su máximo exponente de Lyapunov es positivo. Los sistemas caóticos se caracterizan por las diferentes dinámicas que presentan condiciones iniciales infinitesimalmente próximas, de manera que un sistema dinámico determinista pero caótico aparentemente funciona igual que uno aleatorio, sin serlo. Por este motivo el caos ha supuesto una nueva vía de estudio en la dinámica económica de sistemas, ya que no es necesaria la introducción de perturbaciones aleatorias para conseguir un comportamiento tan irregular y complejo como el observado en la realidad.

Por los motivos enunciados actualmente existen numerosos ejemplos empíricos de cálculo de exponentes de Lyapunov en series temporales procedentes del mundo económico o financiero. Como ejemplos pueden citarse trabajos para datos de tipos de cambio: (Dechert y Gencay, 1992; Bask y de Luna, 2002; Bask, 2002; Weston y Premachandran, 2004), para agregados monetarios: (Serletis, 1995; Barnett et al., 1995), para rendimientos financieros: (Abhyankar et al., 1997; y Shintani y Linton, 2004), para datos bursátiles: (Wang, Chen y Lü, 2004) y para datos reales de producción: (Shintani y Linton, 2003).

En el presente trabajo se plantea analizar la incidencia de decisiones políticas y/o económicas en el ámbito del proceso de Integración Monetaria Europea sobre la estabilidad de los tipos de cambio en el período 1997-2002. De esta manera, en la primera sección se introduce la definición de exponente de Lyapunov y cómo se calcula para series temporales, presentándose los resultados empíricos en la segunda sección. 


\section{CARACTERIZACión DE la Estabilidad DEL Sistema MEdiante EL MÁXIMO EXPONENTE DE LYAPUNOV}

\subsection{Introducción}

Consideramos primero un sistema dinámico unidimensional, en el que trabajamos con dos puntos $x$ e $y$ del espacio de estados $X \in \mathrm{R}$, tan próximos como deseemos, siendo $d_{0}$ la distancia entre ellos. Aplicamos a estos puntos la acción de un flujo $\phi_{t}$ (o una función $f(x)$ en el caso discreto) y, después de un tiempo $t$ sea $d_{t}$ la distancia entre ellos. La idea base de los exponentes de Lyapunov parte de aproximar mediante una exponencial $e^{\lambda_{t} t}$ el cociente de estas distancias, para medir lo que se llama el crecimiento exponencial

$$
\frac{d_{t}}{d_{0}} \approx e^{\lambda_{t} t}
$$

Si para $t \rightarrow \infty$ se puede ver que $\lambda_{t}$ converge a un límite $\lambda$, diremos que $\lambda$ es el exponente de Lyapunov. Además, si este límite es mayor (estrictamente) que cero, podemos decir que bajo la acción del flujo (o de la función) órbitas inicialmente próximas divergen de forma exponencial conforme transcurre el tiempo o, lo que es lo mismo, que el sistema es inestable.

Consideramos el caso discreto. Partimos de un sistema dinámico

$$
x_{k+1}=f\left(x_{k}\right)
$$

siendo $x \in X \subset \mathrm{R}, f: X \rightarrow \mathrm{R}$. Sea una condición inicial $x_{0}$ y su correspondiente órbita $\left\{x_{0}, x_{1}, x_{3}, \ldots\right\}$. Considerando un desplazamiento infinitesimal con respecto a $x_{0}$ dado por el vector $y_{0}$. Entonces se verifica que

$$
f\left(x_{0}+y_{0}\right)=f\left(x_{0}\right)+f^{\prime}\left(x_{0}\right) y_{0}
$$

por lo que, siendo $y_{1}=f\left(x_{0}+y_{0}\right)-f\left(x_{0}\right)$, se cumple que

$$
y_{1}=f^{\prime}\left(x_{0}\right) y_{0}
$$

Siguiendo el mismo planteamiento, la evolución dinámica del vector $y_{n}$ (ver Figura 1) vendrá dada por

$$
y_{n+1}=f^{\prime}\left(x_{n}\right) \cdot y_{n}
$$

con lo cual tenemos la evolución de la distancia entre la órbita original $\left\{x_{0}, f\left(x_{0}\right), f^{2}\left(x_{0}\right), \ldots\right\}$ y la nueva órbita generada a partir de una condición arbitrariamente próxima $\left\{x_{0}+y_{0}, f\left(x_{0}+y_{0}\right), f^{2}\left(x_{0}+y_{0}\right), \ldots\right\}$. 
El factor de crecimiento (o decrecimiento) de este desplazamiento infinitesimal viene dado por el cociente

$$
\frac{\left\|y_{n}\right\|}{\left\|y_{0}\right\|}
$$

y hay crecimiento si $\left\|y_{n}\right\|>\left\|y_{0}\right\|$ o decrecimiento si $\left\|y_{n}\right\|<\left\|y_{0}\right\|$. Aplicando la regla de la cadena tenemos que

(7) $\left.\quad y_{n}=f^{n}\right)\left(x_{0}\right) \cdot y_{0}$

donde $\left.f^{n}\right) \cdot\left(x_{0}\right)=f^{\prime}\left(x_{n-1}\right) \cdot f^{\prime}\left(x_{n-2}\right) \cdot \ldots \cdot f^{\prime}\left(x_{0}\right)$, por lo que $\left.\frac{\left\|y_{n}\right\|}{\left\|y_{0}\right\|}=\mid f^{n}\right)^{\prime}\left(x_{n}\right) \mid$.

FIGURA 1

EVOLUCIÓN DE LA DISTANCIA ENTRE DOS ÓRBITAS GENERADAS A

PARTIR DE CONDICIONES INICIALES ARBITRARIAMENTE CERCANAS

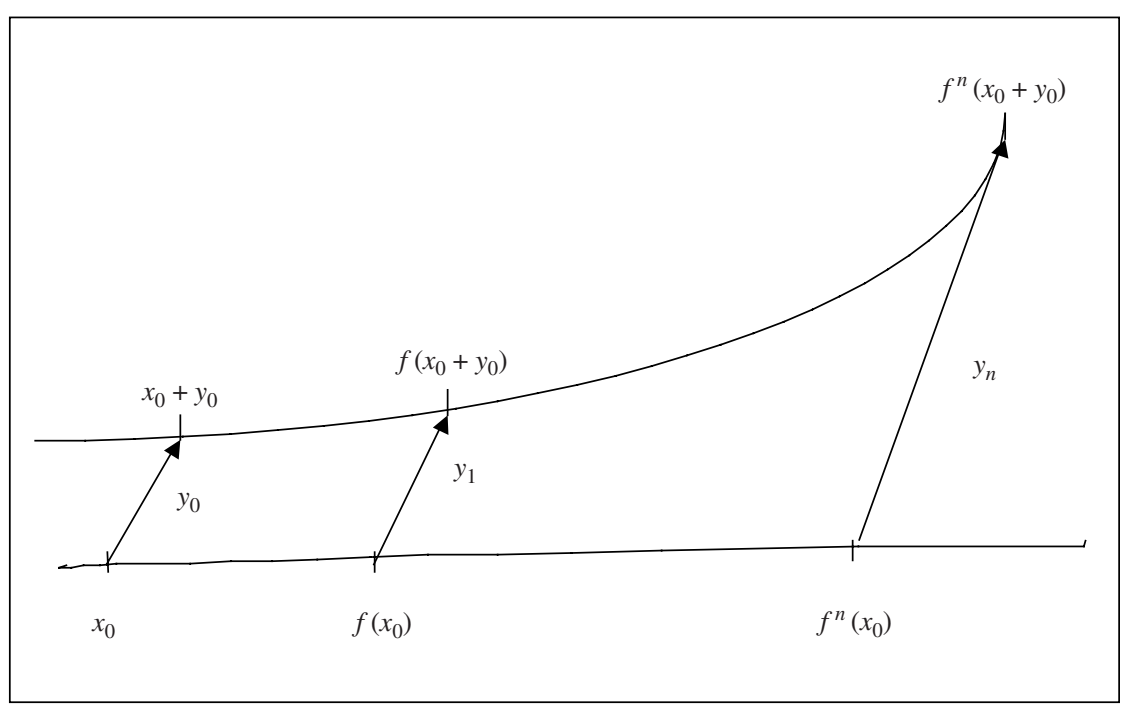

El exponente de Lyapunov será, por tanto, el límite del logaritmo de ese cociente cuando el número de iteraciones sea suficientemente grande, es decir,

(8) $\left.\lambda\left(x_{0}\right)=\lim _{n \rightarrow \infty} \frac{1}{n} \ln \left[\mid f^{n}\right) \cdot\left(x_{n}\right) \mid\right]$ 
Cuando el sistema objeto de estudio es $k$-dimensional, el análisis de la estabilidad en el sentido de Lyapunov se generaliza del valor de la derivada de la función al valor de la matriz jacobiana asociada a dicho sistema dinámico. Recordemos que la matriz jacobiana es la formada por las derivadas parciales con respecto a todas las variables de estado, es decir,

$$
D f=\left(\begin{array}{cccc}
\partial f_{1} / \partial x_{1} & \partial f_{1} / \partial x_{2} & \cdots & \partial f_{1} / \partial x_{k} \\
\partial f_{2} / \partial x_{1} & \partial f_{2} / \partial x_{2} & \cdots & \partial f_{2} / \partial x_{k} \\
\vdots & \vdots & \ddots & \vdots \\
\partial f_{k} / \partial x_{1} & \partial f_{k} / \partial x_{2} & \cdots & \partial f_{k} / \partial x_{k}
\end{array}\right)
$$

Cuando el sistema es $k$-dimensional se debe generalizar la definición de exponente de Lyapunov, sustituyendo la derivada de la función por la matriz jacobiana o, más exactamente, por los autovalores de la misma, de manera que en lugar de hablar de exponente de Lyapunov hablamos de espectro de exponentes de Lyapunov, ya que habrá tantos exponentes como dimensión tenga el sistema. El sistema será inestable si el máximo exponente es positivo.

El $j$-ésimo exponente de Lyapunov para una condición inicial $x_{0}$ se define como

$$
\lambda_{j}\left(x_{0}\right)=\lim _{n \rightarrow \infty} \frac{1}{2 n} \ln H_{n j}
$$

para $j=1, \ldots, k$ siendo $H_{j n}$ los autovalores de la matriz $H_{n}\left(x_{0}\right)=$ $\left(D f^{n}\left(x_{0}\right)\right)^{\prime} D f^{n}\left(x_{0}\right)$, donde $D f^{n}\left(x_{0}\right)=D f\left(x_{n-1}\right) \cdot D f\left(x_{n-2}\right) \cdot \ldots \cdot D f\left(x_{0}\right)$.

\subsection{Cálculo del Exponente de Lyapunov en Series Temporales}

En economía no son conocidos los sistemas dinámicos que rigen el comportamiento de las variables económicas, pero sí se dispone de series temporales, que se supone que son observaciones o realizaciones de estos sistemas desconocidos, denominados sistemas generadores de datos. Por consiguiente, dado que generalmente no se conoce el modelo generador de datos, los exponentes de Lyapunov habrán de ser calculados no a través del sistema sino de la serie generada.

En los últimos tiempos, la estimación de los exponentes de Lyapunov a partir de una serie temporal ha experimentado un impulso de la mano de la aplicación de la metodología de redes neuronales. Tradicionalmente han existido dos métodos para la estimación de los exponentes de Lyapunov a partir de datos: el método directo y el método indirecto o del jacobiano. El primero fue propuesto por Hahn (1963) siendo Wolf (1985) el que generalizó su uso y el segundo por Eckmann y Ruelle (1985). 
Los métodos directos tratan de estimar los exponentes de Lyapunov a partir de su propia definición, midiendo empíricamente la separación de las órbitas generadas a partir de puntos inicialmente próximos. En concreto, se calculan analizando la evolución en el espacio de fases de una elipsoide de condiciones iniciales. Cada exponente mide la tasa media de crecimiento exponencial a largo plazo de cada uno de los ejes del elipsoide y el máximo exponente de Lyapunov marcará la máxima tasa de divergencia de manera que será el más sencillo de calcular. En condiciones ideales (Brock, 1986; Eckmann y Ruelle, 1992), con infinitos datos y sin ningún ruido, el valor proporcionado converge al verdadero valor del exponente máximo de Lyapunov.

Sin embargo, en la realidad las series no son, obviamente, infinitas (es más, en economía las series disponibles suelen ser excesivamente cortas) y además suelen estar perturbadas por factores aleatorios exógenos (ruido). La presencia de ruido afecta muy negativamente a la estimación utilizando estos métodos ya que, en presencia de ruido, pueden proporcionarse exponentes espurios positivos, al incrementarse la divergencia entre las condiciones iniciales consideradas.

En estos casos surge la necesidad de la aplicación de otros métodos robustos a la presencia de ruido. Estos métodos no analizan directamente la evolución seguida por dos condiciones iniciales arbitrariamente próximas, buscando una estimación directa de la tasa de divergencia (o convergencia), sino que estiman esta tasa de divergencia indirectamente, basándose en una estimación previa de la matriz jacobiana (la matriz de derivadas parciales) del sistema subyacente desconocido, generador de la serie temporal. Por este motivo también se los conoce como métodos de estimación del jacobiano. Difieren unos de otros precisamente en la manera de estimar esta matriz.

En un primer momento, los métodos se basaban en estimación lineal lo$\mathrm{cal}^{1}$. Estos métodos producen buenas estimaciones del exponente de Lyapunov cuando las series son suficientemente largas y limpias de ruido, circunstancias difíciles de conseguir en economía. Además, como muestran McCaffrey et al. (1992) el ajuste lineal genera exponentes espurios cuando la dimensión es elevada, de manera que se hace imprescindible el desarrollo de nuevas técnicas. Este desarrollo pasa, lógicamente, por disminuir las restricciones de la metodología propuesta anteriormente, en especial la referida a la linealidad. Es decir, en lugar de estimar una aproximación lineal al jacobiano, tratan de estimarlo mediante una función no lineal. Estos métodos presentan la ventaja fundamental de ofrecer la posibilidad de estimar los exponentes de Lyapunov a partir de series temporales (aplicando métodos de regresión no paramétrica no lineal), aunque las mismas procedan de sistemas que pueden tener perturbaciones estocásticas, y sin necesidad de abundantes datos.

Uno de los métodos más utilizados en estimación de funciones no lineales es el basado en redes neuronales. Estos métodos, inicialmente propuestos por Nychka et al. (1992) y Gencay y Dechert (1992), han funcionado muy bien en la

1 Ver Eckman y Ruelle, 1985, Eckman et al., 1985, Sano y Sawada, 1985, Sato et al., 1987 y Abarbanel et al., 1991, entre otros. 
práctica con series cortas y con ruido, y asimismo han mostrado resultados favorables en simulación como se puede ver en Gallant y White (1992), Kuan y White (1994) y Barnett et al. (1995).

Por último, también muestran buenas propiedades teóricas (ver Nychka et al., 1992; McCaffrey, 1991, y McCaffrey et al., 1992), fundamentalmente su buen nivel de aproximación y la escasa sensibilidad al aumento de la dimensión del sistema. La propiedad de aproximación universal también se aplica a sus derivadas (Gallart y White, 1992), propiedad interesante en el contexto de los exponentes de Lyapunov. Los más utilizados han sido los programados por Ellner et al. (1992) llamado Lyapunov Exponents Neural NetS (LENNS) y Kuan et al. (1997) NETLE 3.01. En ambos casos se utiliza para la estimación del jacobiano el método de mínimos cuadrados no lineales, y se trabaja con una red neuronal SLFFN de una sola capa, trabajando con un número amplio de número de nodos y de inputs, eligiendo el mejor ajuste conseguido en función de la minimización del error cometido.

Adicionalmente, en los últimos tiempos se ha estado trabajando en el desarrollo de la distribución asintótica de probabilidad de los exponentes de Lyapunov calculados mediante redes neuronales. En concreto, Shintani y Linton (2003 y 2004) han probado que convergen asintóticamente a la distribución normal. La obtención de esta distribución es fundamental para extraer conclusiones fiables acerca de la estabilidad o inestabilidad del sistema generador de la serie temporal.

Se parte de una sucesión de escalares aleatorios $\left\{x_{t}\right\}_{t=1}^{T}$ generada por el siguiente modelo no lineal autorregresivo $x_{t}=f\left(x_{t-1}, \ldots, x_{t-d}\right)+u_{t}$ donde $f: \mathrm{R}^{d} \rightarrow \mathrm{R}$ es una función no lineal y $\left\{u_{t}\right\}_{t=1}^{T}$ es una sucesión de perturbaciones aleatorias con las hipótesis habituales. Este modelo puede ser expresado como $Z_{t}=F\left(Z_{t-1}\right)+U_{t}$ donde $Z_{t}=\left(x_{t}, \ldots, x_{t-d+1}\right)^{\prime} \in \mathrm{R}^{d}, f: \mathrm{R}^{d} \rightarrow \mathrm{R}^{d}$ y $U_{t}=\left(u_{t}, 0, \ldots, 0\right)^{\prime}$. El máximo exponente de Lyapunov del sistema dinámico está definido por

$$
\lambda \equiv \lim _{M \rightarrow \infty} \frac{1}{2 M} \ln v_{1}\left(\mathrm{~T}_{M}^{\prime} \mathrm{T}_{M}\right) \quad \mathrm{T}_{M}=\prod_{t=1}^{M} J_{M-t}=J_{M-1} \cdot \ldots \cdot J_{0}
$$

donde $v_{1}(A)$ es el máximo autovalor de una matriz $A$ y $J_{t}$ es la matriz jacobiana de la función $F$ evaluada en $Z_{t}$. Específicamente,

$$
J_{t}=\left[\begin{array}{ccccc}
\Delta f_{1 t} & \Delta f_{2 t} & \ldots & \Delta f_{d-1, t} & \Delta f_{d t} \\
1 & 0 & 0 & 0 & 0 \\
0 & 1 & 0 & 0 & 0 \\
\vdots & \vdots & \ddots & \vdots & \vdots \\
0 & 0 & 0 & 1 & 0
\end{array}\right]
$$

para $t=0,1, \ldots, T-1$, donde $\Delta f_{j t}=D^{e_{j}} f\left(Z_{t}\right)$ para $j=1, \ldots, d$ siendo $e_{j}$ el $j$-ésimo vector elemental $e_{j}=(0, \ldots, 1, \ldots, 0) \in \mathrm{R}^{d}$. 
Si $\hat{f}(\cdot)$ es el estimador de $f(\cdot)$ basado en redes neuronales, sustituyendo este estimador en la fórmula de la matriz jacobiana, se obtiene el estimador de la misma que se denota por $\hat{J}_{t}$. El estimador por redes neuronales del exponente de Lyapunov se define entonces como

$$
\hat{\lambda}_{M}=\frac{1}{2 M} \ln v_{1}\left(\hat{\mathrm{T}}_{M}^{\prime} \hat{\mathrm{T}}_{M}\right) \quad \hat{\mathrm{T}}_{M}=\prod_{t=1}^{M} \hat{J}_{M-t}=\hat{J}_{M-1} \cdot \ldots \cdot \hat{J}_{0}
$$

donde $M$ es el número de puntos utilizados para la estimación del exponente.

\subsection{Redes Neuronales y Estimación del Máximo Exponente de Lyapunov}

Las redes neuronales (ver, por ejemplo, Nychka et al., 1992; Jungeilges, 1996 y White, 1989) son una clase de modelos no lineales inspirados por la arquitectura neuronal básica del cerebro humano. Reflejan la idea de un proceso de aprendizaje entendido como un procedimiento estadístico de tipo recursivo. Son modelos de tipo input-output en los que la función que relaciona la entrada con la salida está determinada por una estructura neuronal. Estos métodos están siendo aplicados a numerosas áreas tan diferentes como la psicología, la computación, la genética, la lingüística y la ingeniería debido a su capacidad para resolver una gran diversidad de problemas como la decodificación de lenguajes, reconocimiento de caracteres escritos a mano, decodificación de sucesiones genéticas proteicas... en definitiva, aplicando este tipo de técnicas se resuelven problemas relacionados con el reconocimiento de patrones, clasificación, detección de no linealidad y predicción no lineal.

En el caso que nos ocupa, la estimación de los exponentes de Lyapunov, podemos decir que gracias a este tipo de modelos se puede aproximar funciones continuas en espacios finito dimensionales. Por consiguiente, es un método que proporciona una aproximación global a la función. Concretamente los autores proponen la utilización de una red simple (la información sólo fluye en un sentido y no existe retroalimentación) con una única capa oculta y una única salida. Este tipo de red neuronal se suele denotar como SLFFN (single-layerfeedforward network) y se muestra en la Figura 2, para dos valores de entrada: $x_{1}$ y $x_{2}$.

Su funcionamiento es el siguiente: las unidades de entrada transforman linealmente los inputs determinada por los denominados factores de intensidad asociado a la conexión entre el nodo input $j$ y el nodo oculto $i$. Así, el input total que entra en el i-ésimo nodo oculto suponiendo d inputs $x$ es $\sum_{j=1}^{d} \chi_{i j} x_{j}$. Cada uno de estos nodos ocultos lleva a cabo una transformación no lineal sobre el input total que le entra, aplicándole una función de activación y produciendo el output

$$
o_{i}=\psi\left(\sum_{j=1}^{m} \chi_{i j} x_{j}+\chi_{i 0}\right) \quad i=1, \ldots, k
$$


FIGURA 2

RED NEURONAL SINGLE-LAYER FEED FORWARD NETWORK (SLFFN)

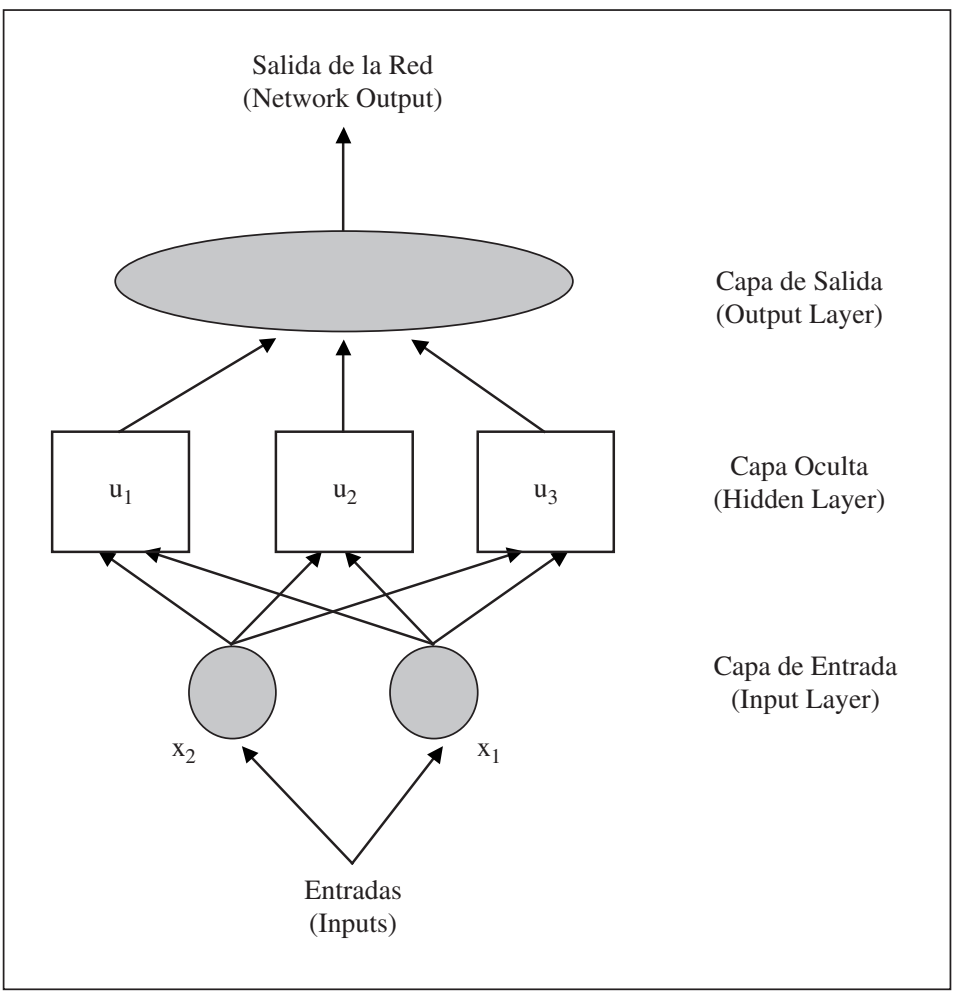

de manera que habrá un output por unidad o nodo en la capa oculta. Esta función de activación es la misma para todas las unidades, pero cada una de ellas tiene su propio sesgo $\chi_{i 0}$ que representa un input externo o el nivel de actividad neuronal intrínseco. Esta función de activación $\psi(u)$ tiene en general forma de ese ${ }^{2}$, con dos asíntotas, en cero cuando $u \rightarrow-\infty$ y en uno cuando $u \rightarrow \infty$. Los autores utilizan la función $\psi(u)=\frac{e^{u}}{1+e^{u}}$.

Los outputs de la capa oculta pasan a través de las unidades de salida que llevan a cabo una transformación sobre su input total. Suponiendo $q$ unidades outputs, la salida de la red será, por tanto

$$
O_{j}=\Phi\left(\beta_{j}+\sum_{s=1}^{k} \beta_{j s} o_{s}\right) \quad j=1, \ldots, q
$$

${ }^{2}$ Se toman funciones de este tipo para modelizar a grandes rasgos las propiedades "umbral" de las neuronas reales. 
En el caso particular de una unidad de salida, el output global se suele considerar como una transformación lineal del input global, de manera que

$$
O=\sum_{i=1}^{k} \beta_{i} \psi\left(\chi_{i}^{\prime} X+\chi_{i 0}\right)
$$

para $k$ unidades en la capa oculta y $d$ inputs, donde $\chi_{i}{ }^{\prime}=\left(\chi_{i 1}, \chi_{i 2}, \ldots, \chi_{i d}\right)$ y $X \in \mathrm{R}^{d}$ es el vector de inputs.

En definitiva, un modelo de redes neuronales general será un modelo que intenta aproximar, mediante la estructura especificada de capas y conexiones, las $q$ unidades de salida $y_{i} i=1, \ldots, q$ mediante funciones no lineales de los $d$ inputs, $f_{i}\left(x_{1}, \ldots, x_{d}\right) i=1, \ldots, q$. Se tratará, por tanto, de estimar el conjunto de ponderaciones y el número de nodos de la capa oculta necesarios para que los valores de entrada, después de pasar por la red, produzcan valores de salida lo más parecidos posible a los que realmente toman las variables. Esta aproximación se realiza en la fase denominada entrenamiento mediante el que, aplicando un proceso recursivo de ajuste mediante minimización del error cometido, la red intenta estimar las relaciones ocultas en los datos.

Según este enfoque y para el caso concreto de la estimación del exponente máximo de Lyapunov, por tanto, la estimación de la función que utilizaremos para estimar la matriz jacobiana y a partir de ella el exponente de Lyapunov máximo será

$$
\hat{f}(X)=\sum_{i=1}^{K} \beta_{i} \psi\left(\chi_{i}{ }^{\prime} X+\chi_{i 0}\right)
$$

donde $\psi(u)=\frac{e^{u}}{1+e^{u}}$ es la función de distribución logística $X=x_{t}^{m} \in \mathrm{R}^{m}$ y $\chi_{i} \in \mathrm{R}^{m}$. Es decir,

$$
x_{t}=\hat{f}\left(x_{t}^{m}\right)=\sum_{i=1}^{K} \beta_{i} \psi\left(\chi_{i 0}+\sum_{j=1}^{m} \chi_{i j} x_{t-j \tau}\right)
$$

donde $m$ y $\tau$ son, respectivamente, la dimensión de absorción y el retardo necesarios para una correcta reconstrucción ${ }^{3}$. Los parámetros se estiman aplicando mínimos cuadrados no lineales dentro de un algoritmo recursivo de minimiza-

\footnotetext{
${ }^{3}$ Esta idea se basa en las nociones de la reconstrucción del atractor que garantiza, gracias al Teorema de Takens (Takens, 1985), la invariabilidad de la dinámica subyacente en el sistema y en su reconstrucción utilizando la serie temporal generada y los parámetros adecuados; en concreto, los exponentes de Lyapunov se mantienen invariantes. La elección de estos parámetros, en concreto, la dimensión de absorción y el retardo, es crítica para la obtención de resultados fiables, desarrollándose gran cantidad de métodos, siendo uno de los más populares la maximización de la bondad de la predicción (Casdagli 1989 y 1992, Sugihara y May, 1990).
} 
ción del error cuadrático medio cometido. Este método de aproximación de la función presenta la ventaja de no ser sensible ante un incremento en la dimensión de absorción. Los autores comprueban que esta estimación es factible para sistemas con ruido con un número escaso de observaciones.

En cuanto al número de nodos de la capa oculta, se trata de la elección más arbitraria en esta metodología. En general, se suele considerar que el número correcto será aquel que minimice el error cometido, evaluando este error por alguno de los métodos habituales.

Por tanto, la principal utilidad de los modelos de redes neuronales referidos al cálculo de los exponentes de Lyapunov se deriva del hecho de su capacidad de aproximar cualquier función no lineal. De hecho, proporcionan la clase de aproximaciones más rica y flexible. Concretamente, como señala White (1989), se ha demostrado que una red SLFNN con una función en forma de ese puede aproximar cualquier función medible con cualquier exactitud (en la métrica apropiada), dado un número suficiente de unidades escondidas.

En el presente trabajo se ha utilizado el algoritmo LENNS (Lyapunov Exponents Neural NetS, 1992). Trabaja con el ajuste de redes neuronales recurrentes de una capa, y el algoritmo de optimización es el estándar BFGS, con el algoritmo BHHH de longitud de paso (Berndt et al., 1974) y el criterio de convergencia sugerido por Gill et al. (1981). Su principal ventaja es la de no restringir la reconstrucción, es decir, que calcula el exponente para un amplio rango de posibles valores de la dimensión de absorción y del retardo, además de los nodos utilizados en la capa de la red (en nuestro caso, las tres variables oscilan entre uno y 10). El exponente de Lyapunov elegido será aquel para el que se maximice la bondad de predicción. Basándose en técnicas de cros-validación, y teniendo en cuenta que se ajustan modelos de redes neuronales a datos generados a partir de sistemas no lineales y ruidosos, se utiliza la minimización del Criterio de Información de Bayes (Nychka et al., 1992; Granger y Teräsvirta, 1992). Básicamente este criterio consiste en penalizar el error cuadrático medio por los parámetros utilizados en el ajuste:

$$
B I C=\frac{1}{2} \cdot\left(1+\ln (2 \cdot \pi)+2 \cdot \ln \left(\frac{1}{N_{m}} \sum_{t} e_{t}^{2}\right)+k \frac{\ln N_{m}}{N_{m}}\right)
$$

donde $k$ es el número de nodos o unidades de la capa oculta, $N_{m}$ es la longitud de la serie de $m$-historias y $e_{t}$ es el error cometido por la red en el instante $t$.

\section{Medición de la Estabilidad de los Tipos de Cambio en el Proceso de Convergencia Europea (1997-2002)}

Se han considerado datos de tipos de cambio con respecto al dólar de las distintas monedas de la Comunidad Económica Europea, esto es, Alemania 
(DEM), Austria (ATS), Bélgica (BEF), Dinamarca (DKK), España (ESP), Finlandia (FIM), Francia (FRF), Gran Bretaña (GBP), Grecia (GRD), Holanda (NLG), Irlanda (IEP), Italia (ITL), Luxemburgo (LUF), Noruega (NOK), Portugal (PTE), Suecia (SEK) y Suiza (CHF). El período de tiempo considerado ha sido desde el 2 de enero de 1997 hasta el 31 de diciembre de 2002 (1504 datos). De estas monedas se han considerado un primer grupo que sólo llega hasta el 31 de diciembre de 1998, constituido por las de los Estados que forman parte de la Unión, con el tipo de cambio fijo y por tanto una misma evolución a partir de este momento. Estos Estados, que cumplían las condiciones de convergencia y que conforman la primera Unión Monetaria son: Alemania, Austria, Bélgica, Finlandia, Francia, Irlanda, Italia, Luxemburgo, Holanda, Portugal y España. Otros dos países que cumplieron los criterios eligieron no participar: Dinamarca y Reino Unido. Y otros dos países no cumplieron los criterios: Grecia (Grecia entró a formar parte de la Unión el 2 de enero de 2001) y Suecia. Por este motivo, y para conseguir un elemento común en toda la serie, se ha considerado adicionalmente la serie del tipo de cambio del euro frente al dólar en todo el período considerado.

Para cada variable se ha subdividido el período 1997-2002, de acuerdo a eventos importantes que podrían haber influido en la evolución de los tipos de cambio mencionados. El Cuadro 1 resume los eventos considerados.

CUADRO 1

EVENTOS CONSIDERADOS PARA EL CORTE DE LAS SERIES

Fecha de corte Evento Observaciones

de la serie

\begin{tabular}{ll}
\hline 17.06.1997 Cumbre de Amsterdam & La cumbre de Amsterdam (16 y 17 de junio \\
& de 1997), con la aprobación del denominado \\
& Pacto de Estabilidad y Crecimiento para dar \\
& credibilidad al euro, limitando el déficit per- \\
& mitido y llegando a acuerdos sobre seguridad, \\
& empleo, política exterior, defensa y legitimi- \\
& dad de las instituciones europeas, que se rati- \\
& ficaron en el Tratado de Amsterdam.
\end{tabular}

4.05.1998 Consejo Europeo El Consejo Europeo de Bruselas (3 de mayo de Bruselas de 1998), en el que se acuerdan los Estados que conformarían la primera Unión Monetaria.

4.01.1999 $\quad \begin{aligned} & \text { Consejo de la Unión } \\ & \text { Europea }\end{aligned}$

El Consejo de la Unión Europea (31 de diciembre de 1998) establece de manera definitiva los tipos de conversión entre el euro y las monedas nacionales de los miembros participantes de la Unión. 


\begin{tabular}{lll}
\hline 27.03.2000 & $\begin{array}{l}\text { Consejo Europeo de } \\
\text { Lisboa }\end{array}$ & $\begin{array}{l}\text { El Consejo Europeo de Lisboa (23 y 24 de } \\
\text { marzo de 2000), en el que, para potenciar el } \\
\text { dinamismo económico en los países de la } \\
\text { Unión, se adoptan diferentes estrategias en } \\
\text { materia de empleo, reforma económica y co- } \\
\text { hesión social. }\end{array}$ \\
\hline 25.09.2000 & $\begin{array}{l}\text { Intervenciones de } \\
\text { Bancos Centrales; } \\
\text { no danés }\end{array}$ & $\begin{array}{l}\text { Intervenciones del Banco Central Europeo, } \\
\text { Reserva Federal de Estados Unidos y Banco } \\
\text { de Japón a favor del euro (septiembre de } \\
\text { 2000). En este mismo mes (28 de septiembre) } \\
\text { Dinamarca rechaza en referéndum su incor- } \\
\text { poración a la zona Euro. }\end{array}$ \\
\hline 7.06.2001 & $\begin{array}{l}\text { No ratificación } \\
\text { por Irlanda }\end{array}$ & $\begin{array}{l}\text { Irlanda, mediante referéndum, no ratifica el } \\
\text { Tratado de Niza (8 de junio de 2000). }\end{array}$ \\
\hline 1.01.2002 & $\begin{array}{l}\text { Entrada en vigor } \\
\text { del euro }\end{array}$ & $\begin{array}{l}\text { Entrada en vigor del euro (1 de enero de 2002) } \\
\text { como moneda única en Bélgica, Alemania, }\end{array}$ \\
& & $\begin{array}{l}\text { Finlandia, Francia, Grecia, Irlanda, Italia, } \\
\text { Luxemburgo, Países Bajos, Austria, Portugal } \\
\text { y España. }\end{array}$ \\
& &
\end{tabular}

Fuente: Elaboración propia.

De esta manera, las series consideradas se han dividido en ocho subperíodos distintos, para así medir la influencia de estos distintos eventos en la estabilidad de los tipos de cambio. Para analizar esta influencia, así como la diferencia entre volatilidad e inestabilidad de los tipos de cambio, se ha calculado, para cada una de estas subseries, el máximo exponente de Lyapunov. A continuación se reflejan los resultados, primero para el período que transcurre desde el 2 de enero de 1997 hasta el 31 de diciembre de 1998 para todas las monedas, y luego para el período completo para las monedas que no entraron en la Unión, el euro y el yen. En los dos casos se proporciona una primera tabla con los exponentes de Lyapunov, y sus respectivas tasas de variación relativa global, y otra segunda tabla con sus variaciones relativas período a período.

TABLA 1

MAXIMO EXPONENTE DE LYAPUNOV ANTES DEL EURO (BLOQUE 1)

\begin{tabular}{lrrrrrrrrrrrr}
\hline \multicolumn{11}{c}{ Exponente de Lyapunov } \\
\hline & ATS & BEF & FRF & DEM & GRD & ITL & ESP & PTE & IEP & FIM & NLG & LUF \\
\hline Per. 1 & 0,096 & 0,155 & 0,129 & 0,085 & 0,041 & 0,077 & 0,183 & 0,022 & 0,123 & 0,075 & 0,080 & 0,155 \\
Per. 2 & 0,053 & 0,071 & 0,074 & 0,063 & 0,031 & 0,075 & 0,056 & 0,084 & 0,102 & 0,079 & 0,063 & 0,055 \\
Per. 3 & 0,057 & 0,020 & 0,072 & 0,027 & 0,512 & 0,035 & 0,150 & 0,062 & 0,041 & 0,048 & 0,019 & 0,046 \\
T.V. & $-0,406$ & $-0,871$ & $-0,442$ & $-0,682$ & 11,49 & $-0,545$ & $-0,180$ & 1,818 & $-0,667$ & $-0,360$ & $-0,763$ & $-0,703$ \\
\hline
\end{tabular}

Nota: Per. corresponde a cada período considerado y T.V. es la tasa de variación relativa global. Fuente: Elaboración propia. 
TABLA 2

MAXIMO EXPONENTE DE LYAPUNOV ANTES DEL EURO (BLOQUE 2)

\begin{tabular}{|c|c|c|c|c|c|}
\hline & \multicolumn{5}{|c|}{ Exponente de Lyapunov } \\
\hline & GBP & DKK & NOK & SEK & CHF \\
\hline Per. 1 & 0,076 & 0,089 & $-4,49$ & $-0,430$ & 0,016 \\
\hline Per. 2 & 0,029 & 0,044 & 0,077 & 0,044 & 0,049 \\
\hline Per. 3 & 0,122 & 0,018 & 0,384 & 0,038 & 0,019 \\
\hline T.V. & 0,605 & $-0,798$ & 1,085 & 1,088 & 0,187 \\
\hline
\end{tabular}

Nota: Per. corresponde a cada período considerado y T.V. es la tasa de variación relativa global. Fuente: Elaboración propia.

TABLA 3

MAXIMO EXPONENTE DE LYAPUNOV (BLOQUE 1)

\begin{tabular}{lrrrrrr}
\hline & \multicolumn{5}{c}{ Exponente de Lyapunov } \\
\hline & GBP & DKK & NOK & SEK & CHF & EURO \\
\hline Per. 1 & 0,076 & 0,089 & $-4,49$ & $-0,430$ & 0,016 & 0,146 \\
Per. 2 & 0,029 & 0,044 & 0,077 & 0,044 & 0,049 & 0,050 \\
Per. 3 & 0,122 & 0,018 & 0,384 & 0,038 & 0,019 & 0,067 \\
Per. 4 & $-0,040$ & $-0,029$ & $-0,047$ & $-0,057$ & $-0,030$ & $-0,027$ \\
Per. 5 & $-0,086$ & 0,026 & 0,039 & 0,087 & 0,194 & 0,026 \\
Per. 6 & $-0,048$ & $-0,025$ & 0,434 & $-0,010$ & $-0,033$ & $-0,025$ \\
Per. 7 & 0,126 & 0,061 & 0,332 & 0,154 & 0,082 & 0,572 \\
Per. 8 & $-0,062$ & $-0,028$ & $-0,020$ & $-0,022$ & $-0,020$ & $-0,028$ \\
T.V. & $-1,816$ & $-1,315$ & 0,995 & 0,948 & $-2,250$ & $-1,192$ \\
\hline
\end{tabular}

Nota: Per. corresponde a cada período considerado y T.V. es la tasa de variación relativa global. Fuente: Elaboración propia.

TABLA 4

VARIACION RELATIVA DEL MAXIMO EXPONENTE DE LYAPUNOV (BLOQUE 2)

\begin{tabular}{lrrrrrr}
\hline & \multicolumn{7}{c}{ Variación Relativa del Exponente de Lyapunov } \\
\hline & GBP & DKK & \multicolumn{1}{c}{ NOK } & SEK & CHF & EURO \\
\hline Per. 1 & $-0,618$ & $-0,506$ & $-1,017$ & $-1,102$ & 2,063 & $-0,658$ \\
Per. 2 & 3,207 & $-0,591$ & 3,987 & $-0,136$ & $-0,612$ & 0,340 \\
Per. 3 & $-1,328$ & $-2,611$ & $-1,122$ & $-2,500$ & $-2,579$ & $-1,403$ \\
Per. 4 & 1,150 & $-1,897$ & $-1,830$ & $-2,526$ & $-7,467$ & $-1,963$ \\
Per. 5 & $-0,442$ & $-1,962$ & 10,128 & $-1,115$ & $-1,170$ & $-1,962$ \\
Per. 6 & $-3,625$ & $-3,440$ & $-0,235$ & $-16,400$ & $-3,485$ & $-23,880$ \\
Per. 7 & $-1,492$ & $-1,459$ & $-1,060$ & $-1,143$ & $-1,244$ & $-1,049$ \\
\hline
\end{tabular}

Nota: Per. corresponde a cada período considerado y T.V. es la tasa de variación relativa global. Fuente: Elaboración propia. 
Lo primero que llama la atención en estas tablas es que ninguno de los países de la Unión que no se incorporaron al euro aumentó su estabilidad a lo largo del período considerado anterior a la entrada del mismo. En concreto, hablamos de Gran Bretaña, Noruega, Dinamarca, Suecia y Suiza, que presentan variaciones relativas positivas del máximo exponente de Lyapunov, salvo en el caso de Dinamarca. Y recordemos que Dinamarca cumplió los denominados 'criterios de convergencia', pero escogió no incorporarse a la zona euro. Es curioso, sin embargo, el caso de Gran Bretaña, que cumplió los criterios de convergencia pero muestra un crecimiento de la inestabilidad en el tipo de cambio, pero concentrado en el tercer período anterior al euro.

En cuanto a los países que se incorporaron a la zona euro, en general mostraron un crecimiento de la estabilidad en el comportamiento de los tipos de cambio en el período anterior al euro, salvo en el caso de Portugal y Grecia. Recordemos, en este sentido, que Grecia cumplió los criterios de convergencia en un momento posterior, mientras que la economía portuguesa, salvo en los últimos tiempos, ha mostrado síntomas de mayor inestabilidad.

Si se considera el período completo 1997-2002 existe una clara diferenciación en el período anterior y posterior al euro. Mientras que antes del euro los máximos exponentes de Lyapunov son positivos, después del euro dichos exponentes pasan a ser, en su mayor parte, negativos. Es decir, que se pasa de un sistema dinámico subyacente inestable a un sistema estable, excluyendo además la posibilidad de existencia de dinámica caótica. Incluso, esto ocurre independientemente de si el país considerado pertenece o no a la zona euro, lo cual indica que este proceso de convergencia y la Unión Monetaria subsiguiente contribuyó a la estabilización económica.

Es curioso además que en el período quinto se observa que los exponentes de Lyapunov para todos los países considerados exceptuando a Gran Bretaña pasan a ser positivos, indicando un claro aumento de la inestabilidad en el sistema dinámico subyacente, que se contrarresta en el período siguiente gracias a la intervención de los Bancos Centrales a favor del euro. Por otro lado, el efecto estabilizador de esta intervención compensa el posible efecto desestabilizador de la negativa danesa a la Unión. El siguiente punto de inflexión se produce con la negativa irlandesa, que nuevamente se traduce en un aumento de inestabilidad del tipo de cambio, siendo de nuevo los máximos exponentes de Lyapunvo positivos. Esta inestabilidad cesa con la entrada en vigor del euro, que refuerza la estabilidad, mostrando otra vez exponentes positivos.

Por último, pero no menos importante, parece claro que a lo largo de todo el período considerado se ha conseguido una clara armonización en las propiedades de estabilidad de los sistemas dinámicos de los países considerados, ya que, comenzando con valores muy diversos del máximo exponente de Lyapunov, los últimos valores observados son todos muy similares, en torno a $-0,02$, excepto en el caso de Gran Bretaña, con $-0,058$ que, de todos modos, sigue siendo un valor bastante próximo al resto. 


\section{Conclusiones}

En el presente trabajo queda demostrada la validez del cálculo de los exponentes de Lyapunov para medir la estabilidad de la evolución de los tipos de cambio en el proceso de convergencia europeo, ya que los resultados observados empíricamente son coherentes con los hechos políticos y económicos reales.

Además, los exponentes de Lyapunov ponen de manifiesto el diferente comportamiento, antes de la creación de la zona euro, de los países que la conformaron y aquellos que no cumplieron los criterios de convergencia, o que decidieron no incorporarse a la zona euro. También se pone de manifiesto el cambio que supuso la creación de la Unión Monetaria para la estabilidad de los tipos de cambio en Europa, ya que pasaron de mostrar valores positivos del máximo exponente de Lyapunov a valores negativos. A lo largo del período considerado se observa un aumento de la estabilidad, además de una clara convergencia en las propiedades dinámicas del sistema subyacente, ya que los valores obtenidos para los exponentes de Lyapunov son similares para los distintos países. Por último, es interesante resaltar el ligeramente distinto comportamiento observado en Gran Bretaña.

\section{REFERENCIAS}

Abarbanel, H.D.I.; R. Brown y M. B. Kennel (1991), Local Lyapunov exponents computed from observed data. Journal of Nonlinear Science 1: 175-199.

Abhayandar, A.; L.S. Copeland y W. Wong (1997), Uncovering nonlinear structure in real-time stock-market indexes: the S\&P 500, the DAX, the Nikkei 225 and the FTSE-100. Journal of Business and Economic Statistics 15: 1-14.

Barnett, W.A.; A.R. Gallant; M.J. Hinich; J.A. Jungeilges; D.T. Kaplan y M.J. Jensen (1995), Robustness of nonlinearity and chaos tests to measurement error, inference method, and sample size. Journal of Economic Behavior and Organization 27: 301-320.

Bask, M. (2002), A positive Lyapunov exponent in Swedish exchange rates? Chaos, Solitons and Fractals 14: 1295-1304.

Bask, M. y X. de Luna (2002), Characterizing the degree of stability of nonlinear dynamic models. Studies in Nonlinear Dynamics \& Econometrics 6, 1: art. 3.

Berndt, E.K.; G.H. Hall; R.E. Hall y J.A. Hausman (1974), Estimation and inference in nonlinear structural models. Annals of Economics and Social Measurement 3/4: 653-665.

Besné, R.; J.R. Canedo; B. Pérez (1998), La Unión Europea: historia, instituciones y sistema jurídico. Bilbao, Universidad de Deusto.

Brock, W.A. (1986), Distinguishing random and deterministic systems. abridged version. Journal of Economic Theory 40: 168-195.

Casdagli, M. (1989), Nonlinear prediction of chaotic time series. Physica D 35: 335-356.

Casdagli, M. (1992), Chaos and deterministic versus stochastic modelling. Journal of the Royal Statistical Society B 54, 2: 303-328.

Dechert, W.D. y R. Gencay (1992), Lyapunov exponents as a nonparametric diagnostic for stability analysis. Journal of Applied Econometrics 7: S41-S60. 
Eckman, J.-P y D. Ruelle (1985), Ergodic theory of chaos and strange attractors. Reviews of Modern Physics 57, 3: 617-656.

Eckman, J.-P. y D. Ruelle (1992), Fundamental limitations for estimating dimensions and Lyapunov exponents in dynamical systems. Physica D 56: 185-187.

Ellner, S.; D.W. Nychka y A.R. Gallant (1991), LENNS, a program to estimate the dominant Lyapunov exponent of noisy nonlinear system from time series data. Institute of Statistics Mimeo Series \#2235, Statistics Department, North Carolina State University, Raleigh, NC 27695-8203.

Gallant, A.R. y H. White (1992), On learning the derivatives of an unknown mapping with multilayer feedforward networks. Neural Networks 5: 12-138.

Gencay, R. y W.D. Dechert (1992), An algorithm for the $n$ Lyapunov Exponents of an $n$ dimensional unkown dynamical system. Physica D 59: 142-157.

Gill, P.E.; W. Murray y M.H. Wright (1981), Practical Optimization. London y New York. Academic Press.

Granger, C.W.J. y T. Teräsvirta (1992), Experiments in modeling nonlinear relationships between time series. En Nonlinear modelling and forecasting (Eds. M. Casdagli y S. Eubank). Santa Fe Institute Studies in the Sciences of Complexity, Proceedings v. XII. New York. Addison-Wesley: 199-226.

Jungeilges, J.A. (1996), Operational characteristics of White's test. En Nonlinear dynamics and economics (Eds. W.A. Barnett, A.P. Kirman y M. Salmon). Cambridge. Cambridge University Press: 219-266.

Kuan, C.M.; T. Liu y R. Gencay (1997), Netle 3.01: feedforward neural networks and Lyapunov exponents estimation. Ball State University.

Kuan, C.M. y H. White (1994), Artificial neural networks. an econometric perspective. Econometric Reviews 13: 1-91.

Lyapunov, A.M. (1892), The general problem of the stability of motion. Mathematical Society of Kharkov. PhD. Traducido en International Journal of Control (1992) 55: 131-773.

McCaffrey, D.F.; S. Ellner; A.R. Gallant y D.W. Nychka (1992), Estimating the Lyapunov exponent of a chaotic system with nonparametric regression. Journal of the American Statistical Association 87: 682-695.

Nychka, D.; S. Ellner; A.R. Gallant y D. McCaffrey (1992), Finding chaos in noisy systems; Journal of the Royal Statistical Society B. 54, 2: 399-426.

Sano, M. y Y. Sawada (1985), Measurement of the Lyapunov Spectrum from a chaotic time series. Physical Review Letters 55, 10: 1082-1085.

Sato, S.; M. Sano e Y. Sawada (1987), Practical methods of measuring the generalizad dimension and the largest Lyapunov exponent in high dimensional chaotic systems. Progress of Theoretical Physics 77, 1: 1-5.

Serletis, A. (1995), Random walks, breaking trend functions, and the chaotic structure of the velocity of money. Journal of Business and Economic Statistics 13: 453-458.

Shintani, M. y O. Linton (2003), Is there chaos in the world economy? A nonparametric test using consistent standard errors. International Economic Review 44, 1: $331-358$.

Shintani, M. y O. Linton (2004), Nonparametric neural networkd estimation of Lyapunov exponents and a direct test of chaos. Journal of Econometrics 120: 1-33.

Sugihara, G. y R.M. May (1990), Nonlinear forecasting as a way of distinguishing chaos from measurement error in time series. Nature 334: 734-741. 
Takens, F. (1986), Detecting strange attractors in turbulence en D.A. Rand y L.-S. Young (eds.): Lecture Notes in Mathematics: Dynamical Systems and Turbulence 89; New York, Springer-Verlag: 366-381.

Tamames, R. (1998), Unión Monetaria y el Euro: la recta final. Madrid, Espasa Calpe.

Wang, H.; G. Chen y J. Lü (2004), Complex dynamical behaviors of daily data series in stock exchange. Physics Letters A 333: 246-255.

White, A. (1989), Some asymptotic results for learning in single hidden-layer feedforward network models. Journal of the American Statistical Association 84, 408: 1003-1013.

Wolf, A.; J.B. Swift; H.L. Swinney y J.A. Vastano (1985), Determining Lyapunov exponents from a time series. Physica D 16: 285-317.

Weston, R. y P. Premachandra (2004), A chaotic analysis of the New Zealand exchange rate 1985-2004. NZAE 2004. 